\title{
Probing the radiative electromagnetic local density of states in nanostructures with a scanning tunneling microscope
}

Shuiyan Cao, ${ }^{\dagger,}, @$ Mario Zapata-Herrera, $, \S, @$ Alfredo Campos,,$\perp$ Eric Le Moal,,$^{\ddagger}$ Sylvie Marguet,\# Gérald Dujardin, "Mathieu Kociak, Javier Aizpurua," Andrei G. Borisov, ${ }^{\ddagger}$ and Elizabeth Boer-Duchemin ${ }^{*, \ddagger}$

$\dagger$ Department of Applied Physics, Nanjing University of Aeronautics and Astronautics, Nanjing, China

$\ddagger$ Université Paris-Saclay, CNRS, Institut des Sciences Moléculaires d'Orsay (ISMO), 91405 Orsay, France

IMaterials Physics Center CSIC-UPV/EHU and Donostia International Physics Center DIPC, 20018 Donostia-San Sebastián, Spain

§Present address: CIC nanoGUNE, 20018 Donostia-San Sebastián, Spain and Universidad Distrital Francisco José de Caldas, Bogotá, Colombia

|| Université Paris-Saclay, CNRS, Laboratoire de Physique des Solides (LPS) 91405 Orsay, France

$\perp$ Present address: Facultad de Ciencias y Tecnología, Universidad Tecnológica de Panamá, Panama

\#Université Paris-Saclay, CEA, CNRS, NIMBE, CEA Saclay, Gif-sur-Yvette, France @ Contributed equally to this work

E-mail: Elizabeth.Boer-Duchemin@u-psud.fr 


\begin{abstract}
A novel technique for the investigation of the radiative contribution to the electromagnetic local density of states is presented. The inelastic tunneling current from a scanning tunneling microscope (STM) is used to locally and electrically excite the plasmonic modes of a triangular gold platelet. The radiative decay of these modes is detected through the transparent substrate in the far field. Emission spectra, which depend on the position of the STM excitation, as well as energy-filtered emission maps for particular spectral windows are acquired using this technique. The STM-nanosource spectroscopy and microscopy results are compared to those obtained from spatially resolved electron energy loss spectroscopy (EELS) maps on similar platelets. While EELS is known to be related to the total projected electromagnetic local density of states, the light emission from the STM-nanosource is shown here to select the radiative contribution. Full electromagnetic calculations are carried out to explain the experimental STM data, and provide valuable insight into the radiative nature of the different contributions of the breathing and edge plasmon modes of the nanoparticles. Our results introduce the STM-nanosource as a tool to investigate and engineer light emission at the nanoscale.
\end{abstract}

\title{
Keywords
}

electromagnetic local density of states, inelastic electron tunneling, electron energy loss spectroscopy, surface plasmon, scanning tunneling microscopy

Increasing the rate or efficiency of elementary quantum processes in light sources is a current challenge. One way to do this is to engineer a fluorophore's emission properties by modifying its surrounding environment, thus influencing the electromagnetic local density of states (EM-LDOS). ${ }^{1,2}$ Manipulating this quantity leads to, for example, improved quantum efficiencies for solar cells, ${ }^{3}$ increased brightness for entangled photon pair emission ${ }^{4}$ or ultrafast sources based on spontaneous emission. ${ }^{5}$ Thus the measurement of the EM-LDOS 
is essential for the design and characterization of future nanophotonic devices, and for the further understanding of the role of the EM-LDOS in the emission of light.

The EM-LDOS is the number of electromagnetic modes at a particular energy existing at a particular point in space, where the contribution of each mode is determined by the intensity of the mode at that point. ${ }^{2,6}$ Most often one considers the projected EM-LDOS which takes into account the mode density only along a certain direction, i.e., that of the orientation of the dipole representing an emitter. The projected EM-LDOS at a given point in space determines the total decay rate $\Gamma$ of a dipole emitter placed at that point, and it can be expressed using the dyadic Green's function. ${ }^{2,7}$ Depending on the decay channels, the projected EM-LDOS may be further divided into the radiative (R-EM-LDOS) and nonradiative (NR-EM-LDOS) electromagnetic density of states, each associated, respectively, with the radiative and non-radiative partial decay rate contributions to $\Gamma .{ }^{2}$ For an emitter placed in the vicinity of a plasmonic nanoparticle, the "bright" plasmon modes of the nanoobject are those which are strongly coupled to the radiation continuum in the far field, thus providing a leading contribution to the R-EM-LDOS. The "dark" modes of the system, on the other hand, mainly contribute to the NR-EM-LDOS.

Since the EM-LDOS is a local property, a nanoscale probe is needed for its measurement. Using electron energy loss spectroscopy (EELS) ${ }^{8-11}$ in a scanning transmission electron microscope (STEM), it has been shown that in most cases a high energy $(\geq 10 \mathrm{keV})$ electron beam passing through a thin sample loses its energy in proportion to the EM-LDOS (thus reflecting both the radiative and non-radiative contributions) projected along the direction of the electron beam. ${ }^{12,13}$ The lateral component may be determined from the lateral deflection of the inelastically scattered electrons. ${ }^{14}$ In a related technique, cathodoluminescence, where again a high-energy electron beam is used for excitation, the resulting emitted light is detected and it is the projected R-EM-LDOS that is probed, ${ }^{15-18}$ though dark modes may also be detected through a combination of methods. ${ }^{19}$ Other techniques for mapping the EM-LDOS are based on the use of fluorescent probes, ${ }^{20-27}$ two-photon luminescence ${ }^{28,29}$ 
and/or scanning near-field optical microscopy. ${ }^{30-32}$ Photoelectron emission microscopy is also a remarkable technique for imaging optical near-fields. ${ }^{33,34}$

The scanning tunneling microscope (STM) is a well-known tool for mapping the electronic local density of states, ${ }^{35}$ i.e., the number of electronic levels per unit volume in an infinitesimal energy range, which may even be investigated optically in certain cases. ${ }^{36}$ While light was first seen from the STM tunnel junction as early as the late 1980s, ${ }^{37}$ it is not until recently that an "STM-nanosource" has been used systematically to study the properties of surface plasmon polaritons (SPPs) in plasmonic gaps and nanostructures ${ }^{38-45}$ and to locally and electrically activate directional sources of light. ${ }^{46,47}$ Recently, considerable progress has been made towards replacing the STM-nanosource with antenna-enhanced integrated junctions for the efficient electrical excitation of plasmons and light. ${ }^{48-53}$

The electrical excitation of photons and plasmons in the STM is generally attributed to the inelastic tunneling current between the tip and sample. ${ }^{37,52,54}$ While a large majority of the tunneling electrons cross the tunnel barrier elastically, a small percentage (about $1 \%{ }^{54}$ ) does so inelastically, losing part or all of the excess energy in the barrier. This released energy may excite the different modes of the system. Similar to the classical description of quantum emitters, the inelastic tunneling current responsible for this local, electrical excitation of the electromagnetic modes in a nanosystem may be represented as an oscillating point dipole. This dipole is located in the nanocavity formed between the STM tip and the sample, and oriented in the direction of the tunneling current. ${ }^{40,54-56}$ Given this description of the inelastic tunneling current, and the above definition of the EM-LDOS, the question naturally arises whether the STM-nanosource may be used to probe the electromagnetic local density of states. More specifically, does the radiative yield of this nanosource provide a useful tool for determining the radiative component of the EM-LDOS?

In order to answer this question, we perform an experiment where the STM tip is scanned across a triangular gold platelet deposited on an ITO-covered glass substrate. At each point in the scan the emitted radiation is collected in the far field, providing a map of the STM- 
excitation efficiency on the sample (or "radiation map"). We also use spatially resolved electron energy loss spectroscopy (EELS) to obtain maps of the total EM-LDOS. Along with this experimental study, we perform electromagnetic calculations of the EM-LDOS and R-EM-LDOS, leading to maps of these quantities as a function of position on the triangular gold platelet. Similarly, calculations of the emitted light when an STM tip and vertical oscillating dipole are scanned over a triangular gold platelet are performed. This theoretical analysis is further used to unambiguously identify the nature of the plasmonic modes and their contributions to the near and far fields. Our joint experimental and theoretical study shows that the radiation maps obtained with an STM-nanosource are directly related to the R-EM-LDOS. Thus, the scanning tunneling microscope offers an extremely attractive method to locally, and eventually simultaneously, probe the atomic, electronic, and photonic structure of a nano-object.

\section{Results and discussion}

The spatially-resolved studies of the emission of light from an STM-nanosource and the electron energy loss spectroscopy (EELS) experiments are performed on mono-twinned gold crystals of triangular shape, deposited on a substrate (see the Methods section for details of the sample preparation). The substrates are either glass coverslips, $170 \mu \mathrm{m}$ thick, covered with a conducting $85 \mathrm{~nm}$ thick ITO layer (for STM measurements), or $15 \mathrm{~nm}$ thick silicon nitride membranes (for EELS measurements). Figure 1a shows a scanning electron micrograph of such a triangle while Figure 1b shows a three-dimensional projection of an STM topography image of such a structure. The triangles considered here have side lengths $L$ ranging from $600 \mathrm{~nm}$ to $900 \mathrm{~nm}$, and are $20 \mathrm{~nm}$ thick.

The local excitation of the electromagnetic modes at specific locations on the triangular sample is carried out with a commercial STM head mounted on an inverted optical microscope (see Methods and Figure 1c). The inelastic tunneling current between the STM tip 

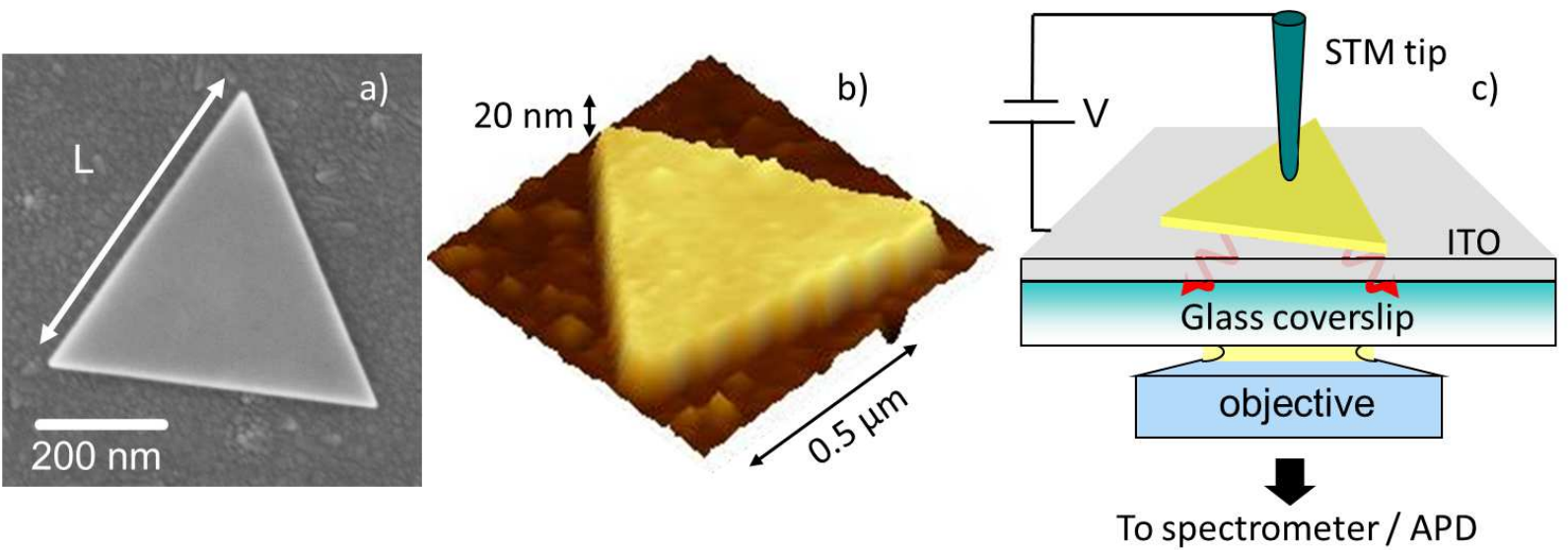

Figure 1: Sample and experimental set-up. (a) Scanning electron micrograph and (b) 3D projection of a scanning tunneling microscopy image of a colloidal, triangular, gold platelet. Typical side lengths $L$ of the studied particles are 600 to $900 \mathrm{~nm}$. The platelets are $20 \mathrm{~nm}$ thick. For the STM image, a voltage of $0.5 \mathrm{~V}$ is applied and the set-point current is $0.1 \mathrm{nA}$ (constant current mode). (c) Schematic of the experimental set-up. The inelastic tunneling current between the STM tip and metal sample excites the plasmonic modes of the triangle platelet which then decay radiatively. The resulting emission is collected using an oil immersion objective of high numerical aperture $(\mathrm{NA}=1.49)$ and focused on either the entrance slit of a spectrometer or on an avalanche photodiode (APD).

and metal sample generates the light which is collected through the transparent substrate using an oil immersion objective of high numerical aperture $(\mathrm{NA}=1.49)$.

The emission of light is associated with the radiative decay of the electromagnetic modes of the system. In order to identify the energies of these modes, we measure the spectra of the emitted light upon excitation with the STM-nanosource (see Figure 2). The STM-nanosource is inherently broad-band ${ }^{38}$ and depends on the applied voltage (leading to a high-energy cut-off) ${ }^{57}$ In order to assure that we excite the inherent resonances of the sample ${ }^{58}$ and not the nanocavity resonances of the tip-sample system, ${ }^{56,59}$ we use a tungsten STM tip (see Methods). Since tungsten is a non-plasmonic metal in the energy range under consideration (i.e., the real part of the permittivity is positive and the spectral response is broad and damped ${ }^{60}$ ) nanocavity resonances are thus avoided (see Section 1 of the Supplementary Information for further details).

Figure 2 shows the resulting experimental spectra of the light emitted when the STMnanosource is placed in the center of the upper facet (Figure 2a, red curve) or in the middle of 

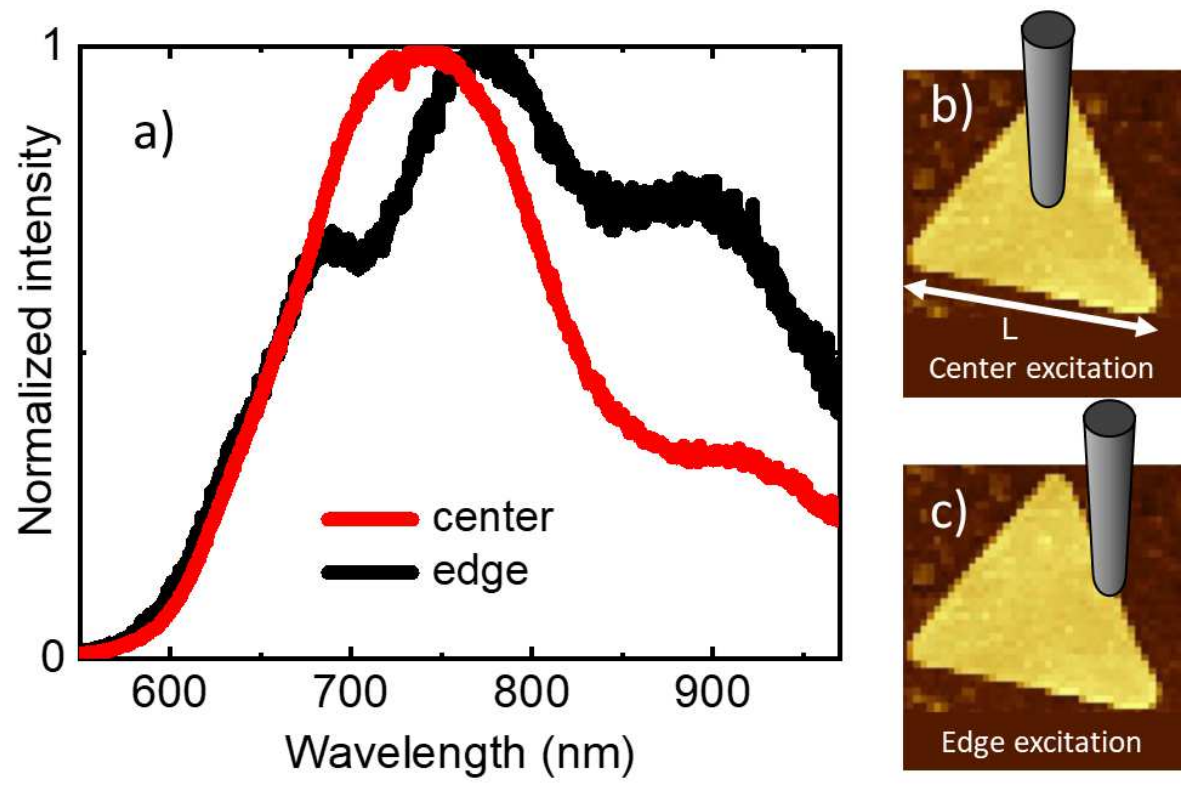

Figure 2: Experimental spectra for different excitation positions. The red curve shows the resulting light emission when the STM tunneling current excites the triangular platelet in the center of its top face, and the black curve shows the data obtained when the excitation is located on the edge of the triangle. Note that the decrease in intensity at short wavelengths $(\lesssim 575 \mathrm{~nm})$ is due to the interband transitions in gold. The triangular platelet side length $L$ is $600 \mathrm{~nm}$. The spectra are normalized by their maximum values. STM parameters are $I_{\text {set-point }}=0.5 \mathrm{nA}$ and $V=2.8 \mathrm{~V}$ and the acquisition time is $300 \mathrm{~s}$. Panels $\mathrm{b}$ and c show schematically the excitation geometries.

the edge (Figure 2a, black curve) of the triangular platelet. (See panels b and c of the figure for a schematic of the tip position). When the STM excitation is located in the center of the upper facet of the triangular platelet, two broad overlapping resonances may be observed in the light emission at about $730 \mathrm{~nm}$ and $910 \mathrm{~nm}$ as obtained from fits to the data. When the excitation is located on the edge of the triangle, three different overlapping resonances are apparent at wavelengths of about $670 \mathrm{~nm}, 760 \mathrm{~nm}$ and $870 \mathrm{~nm}$. These results suggest that (i) the STM tunneling current indeed excites the plasmonic modes of the platelet which then decay radiatively and (ii) different modes may be selectively excited depending on the particular position of the STM tip. ${ }^{46,58}$ We will use these spectral results to select the wavelength windows for the light emission mapping experiments.

Two wavelengths are chosen for the STM-nanosource mapping experiments. In a mapping 
experiment, the STM scans the particle under study in constant current mode $\left(I_{\text {set-point }}=\right.$ $0.1 \mathrm{nA}$ and $V=2.8 \mathrm{~V}$ ). At each pixel in the scan, the topography, tunneling current, and number of photons emitted into the substrate in the collection angle of the objective are recorded (see Supporting Information Section 2 for details). The photodetector is an avalanche photodiode in front of which different bandpass filters are placed so that wavelength-dependent maps may be obtained. The light emission maps are divided by the simultaneously recorded tunnel current signal in order to obtain the excitation efficiency or photon yield (in photons/electron).

Plotting the emitted light per electron as a function of STM excitation position for two different energy ranges yields very different results. In Figure 3a, the number of photons emitted per electron in a wavelength range of $750 \pm 20 \mathrm{~nm}$ as a function of excitation position is shown, while Figure 3b shows the same for a wavelength range of $900 \pm 20 \mathrm{~nm}$. For the higher energy/shorter wavelength range, more light is emitted per electron when the STM tip is located in the center of the upper face of the platelet, while for the lower energy/longer wavelength range, the most efficient excitation of light occurs when the STM tip is located on the edge of the nanoparticle. This striking contrast would suggest that different types of electromagnetic modes are excited in each case.

In order to gain further insight into the character of the plasmonic modes contributing to the emission maps obtained with the STM-nanosource, and to investigate the link between these results and the electromagnetic EM-LDOS, similar experiments are performed using electron energy loss spectroscopy (EELS) (see Methods and Section 3 of the Supporting Information). Looking at the EELS data in Figure 3c and Figure 3d for a similar triangular platelet, we obtain a result that shows similar trends as in the case of the STM-nanosource excitation: considering a high energy/short wavelength range, a strong signal is measured when the excitation (a high energy $(100 \mathrm{keV}$ ) electron beam) is incident on the flat upper surface of the triangle platelet; for a low energy/long wavelength range, the signal is most significant when the excitation is along the triangle edge. 


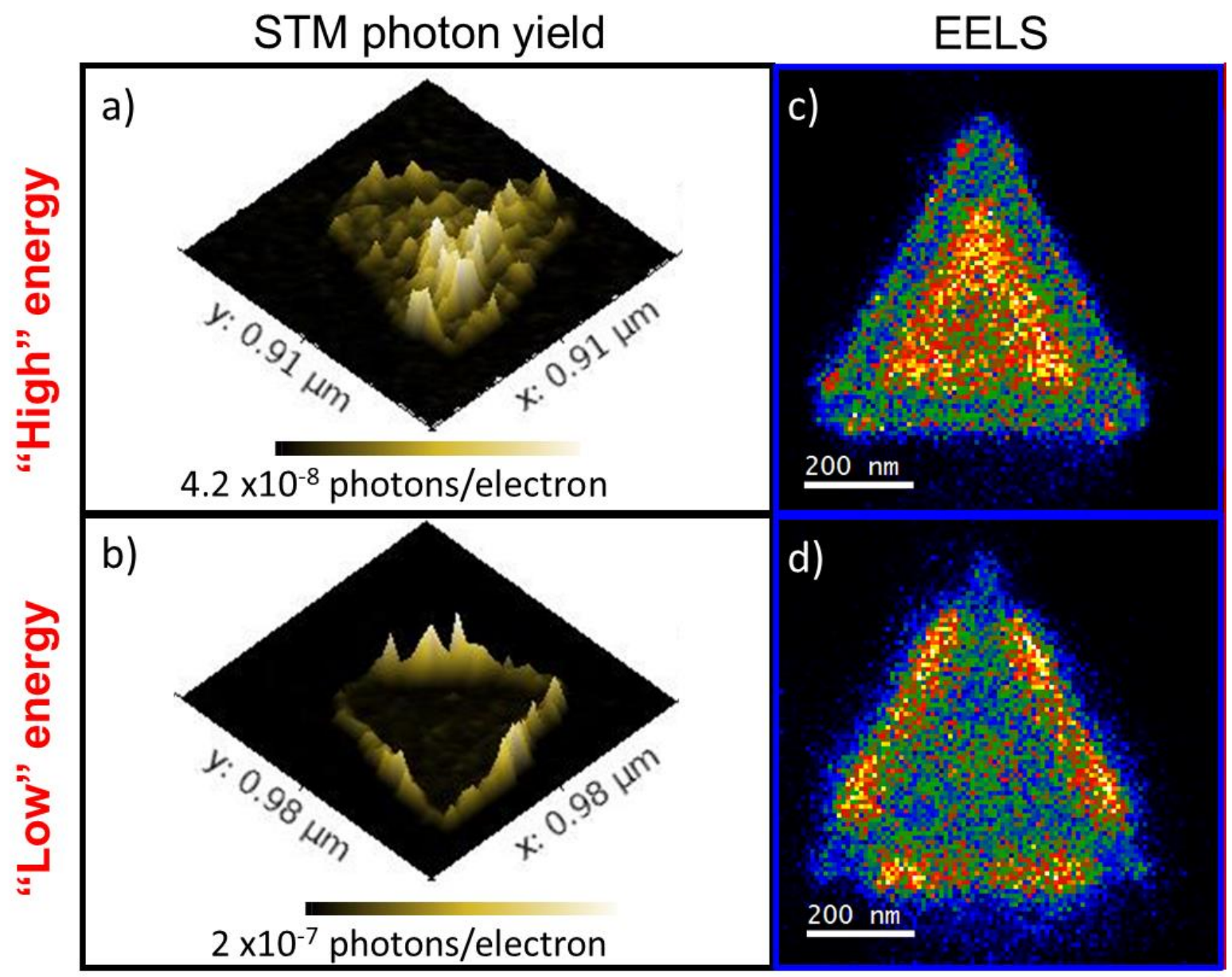

Figure 3: STM-excitation photon yield maps and EELS measurements (a)-(b) These photon yield maps are obtained by dividing the simultaneously acquired photon images by their corresponding current maps (see Section 2 in the Supporting Information for details). The result is thus an efficiency in terms of the number of photons emitted per tunneling electron. In other words, from these results we can predict the best location for the excitation in order to get the most emission at the desired wavelength. In (a), a 750/40 nm bandpass filter is placed before the detector, while in (b), photons in the range $900 \pm 20 \mathrm{~nm}$ are collected. The result is noticeably different for these two wavelength ranges. (c)-(d) Electron energy loss results. A high energy electron beam scans the particle and the energy lost by the electrons passing through the sample is measured. In (c) the number of electrons having lost energy in the 1.5-1.8 eV range as a function of e-beam position is plotted while in (d) the energy loss range is 1.1-1.4 eV. These results match the STM-excitation images qualitatively. The triangle side length is $L \sim 630 \mathrm{~nm}$. 
Since EELS is a loss spectroscopy, it reflects the total EM-LDOS projected along the axis of the incoming electron beam; ${ }^{12}$ i.e., it is equally sensitive to both the dark (i.e., non-radiative) and bright (or radiative) modes of the system. In the STM excitation experiment, however, the emitted light is detected in the far field. This would suggest that the STM-nanosource experiments are only sensitive to the radiative R-EM-LDOS, whose leading contribution is from the bright modes of the sample. The dark modes of the triangular platelet should only contribute to the observed light emission maps if the STM tip or retardation effects allow these modes to couple to the far field. A simulation of the STM experiment is thus necessary to interpret the STM-nanosource results and to further investigate the link between the experimental data and the EM-LDOS.

The first step in the theoretical analysis is the determination of the total and radiative local density of electromagnetic states as well as the electromagnetic (EM) modes of the triangular platelets, so that the nature of the EM modes excited by the tunneling current may be identified. In Figure 4 the calculated wavelength dependence of the EM-LDOS (dashed curves) and R-EM-LDOS (continuous curves) of a triangular platelet is shown for different positions of the excitation point dipole. Here, the R-EM-LDOS is obtained by calculating the emission in all directions when a point dipole with a flat spectrum is placed at different lateral positions $1 \mathrm{~nm}$ above the triangle (see the inset of Figure 4b). Meanwhile, the total EM-LDOS is obtained from the electric field at the position of the dipole. ${ }^{2}$

The resonance peaks in the EM-LDOS and R-EM-LDOS are associated with surface charge density distributions on the sample of specific symmetry (see Figure 4). Based on these symmetry considerations, two main sets of plasmonic modes for the triangular platelet are identified: edge and breathing modes, in accordance with previous classifications in the literature. ${ }^{61-67}$ The edge modes contribute preferentially to the EM-LDOS above the vertices and edges of the triangle (Figure 4a), and the number of nodes in the induced charge density distribution of these modes increases with decreasing wavelength. ${ }^{61-63}$ The low-order modes of this type appear at long wavelengths and include the dipolar plasmon resonance (located 

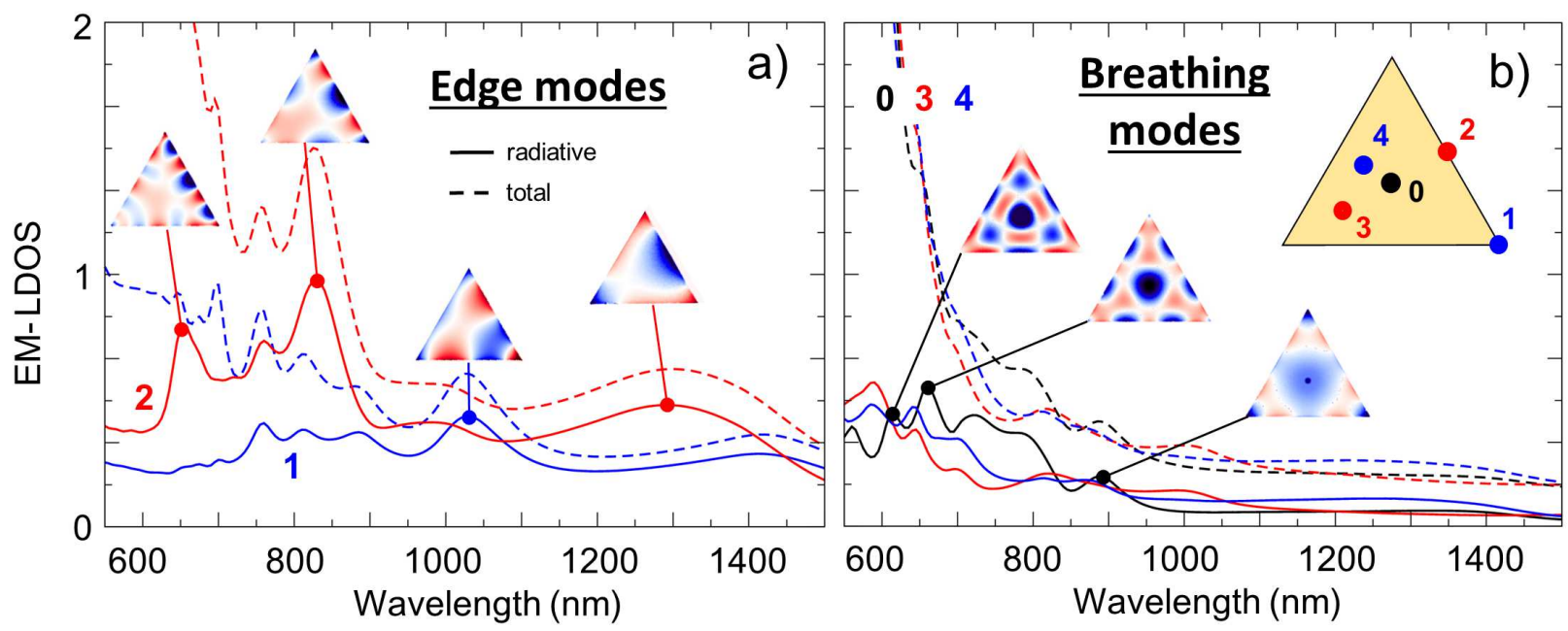

Figure 4: Calculated total local density of electromagnetic states (EM-LDOS, dotted lines), and calculated radiative local density of electromagnetic states (R-EM-LDOS, continuous lines) as a function of wavelength at different positions on the sample. For the sake of comparison the EM-LDOS is scaled by a factor of 1.5 (EM-LDOS/1.5 is shown). (a) EMLDOS and R-EM-LDOS as a function of wavelength at a vertex and on the edge of the platelet. (b) EM-LDOS and R-EM-LDOS as a function of wavelength in and near the center of the platelet. The position at which each curve is calculated is shown in the inset of panel (b). The surface charge densities of the main modes are displayed above their corresponding peaks in the R-EM-LDOS. The triangle side length used in these calculations is $L=650 \mathrm{~nm}$.

above $2500 \mathrm{~nm}$ ), and the quadrupolar plasmon resonance at $1300 \mathrm{~nm}$ (excitation in the center of the edge, point 2). These edge modes are clearly radiative: consider for example the lowest order edge mode for which the charge density distribution is shown in Figure 4a $(\lambda=1300 \mathrm{~nm})$; the radiative $\mathrm{R}$-EM-LDOS is more than half the value of the total EM-LDOS (recall that the total EM-LDOS is divided by a factor of 1.5 in Figure 4).

The second set of EM modes contributes preferentially to the EM-LDOS above the center of the platelet (Figure 4b). In this case, the induced charge distribution is concentric on the platelet facets. This is characteristic of breathing or film-like modes ${ }^{61-66}$ launched on a gold film by a vertical oscillating dipole and discretized by the reflections at the platelet boundaries. By symmetry, these breathing modes have no net induced dipole moment and are in general much less radiative as compared to the edge modes. This again may be seen by comparing the total and radiative EM-LDOS at for example $900 \mathrm{~nm}$ (lowest order breathing 
mode for which the charge density distribution is shown in Figure 4b). In this case, the total EM-LDOS is equal to more than a factor of three times the radiative R-EM-LDOS. The fact that the edge modes are clearly more radiative than the breathing modes (for $\lambda>600 \mathrm{~nm}$ ) is confirmed in Section 4 of the Supporting Information and in Figure 5a below.

At wavelengths of $\lambda \lesssim 800 \mathrm{~nm}$, higher-order plasmonic modes are excited. These higherorder modes involve rapidly oscillating charge densities and higher-order multipole moments, thus reducing the coupling of these modes to the far field. At the same time, however, we see that the total EM-LDOS of the breathing modes diverges for shorter wavelengths (see Figure 4b). Since the total EM-LDOS increases drastically, the radiative R-EM-LDOS increases proportionally. In the competition between these two effects (reduced far-field coupling and the increase in the total number of electromagnetic modes) the increase in the total EM-LDOS dominates, leading to higher values of the radiative R-EM-LDOS at shorter wavelengths as compared to longer wavelengths. In fact, at these shorter wavelengths, the R-EM-LDOS of the breathing modes is comparable to the R-EM-LDOS values of the edge modes (consider for example the continuous blue curves in Figure 4a and Figure 4b).

The connection between the R-EM-LDOS and the experimental spectra shown in Figure 2 is made is Figure 5a. Here we calculate the spectrum of the light emitted into the substrate when the STM-nanosource excites the platelet; as opposed to the previous calculation for the R-EM-LDOS, these results include the STM tip, exclude the emission in the half space above the platelet, and take into account the STM excitation spectrum ${ }^{57}$ (see Methods and Section 5 in the SI). The theoretical results in Figure 5a are obtained for triangles of different side length and for the two relevant tip positions addressed experimentally.

Comparing Figures 4 and 5a, it may be seen that the emission spectra calculated for the complete STM system (platelet plus tip) closely resembles the R-EM-LDOS of the isolated triangular platelet (i.e., no tip included; see also Section 5 in the SI for a plot of the data of Figures 4 and 5 on the same graph). This demonstrates (i) the non-perturbing role of the STM tip as outlined earlier; (ii) the similarity, up to a scaling factor, between the 


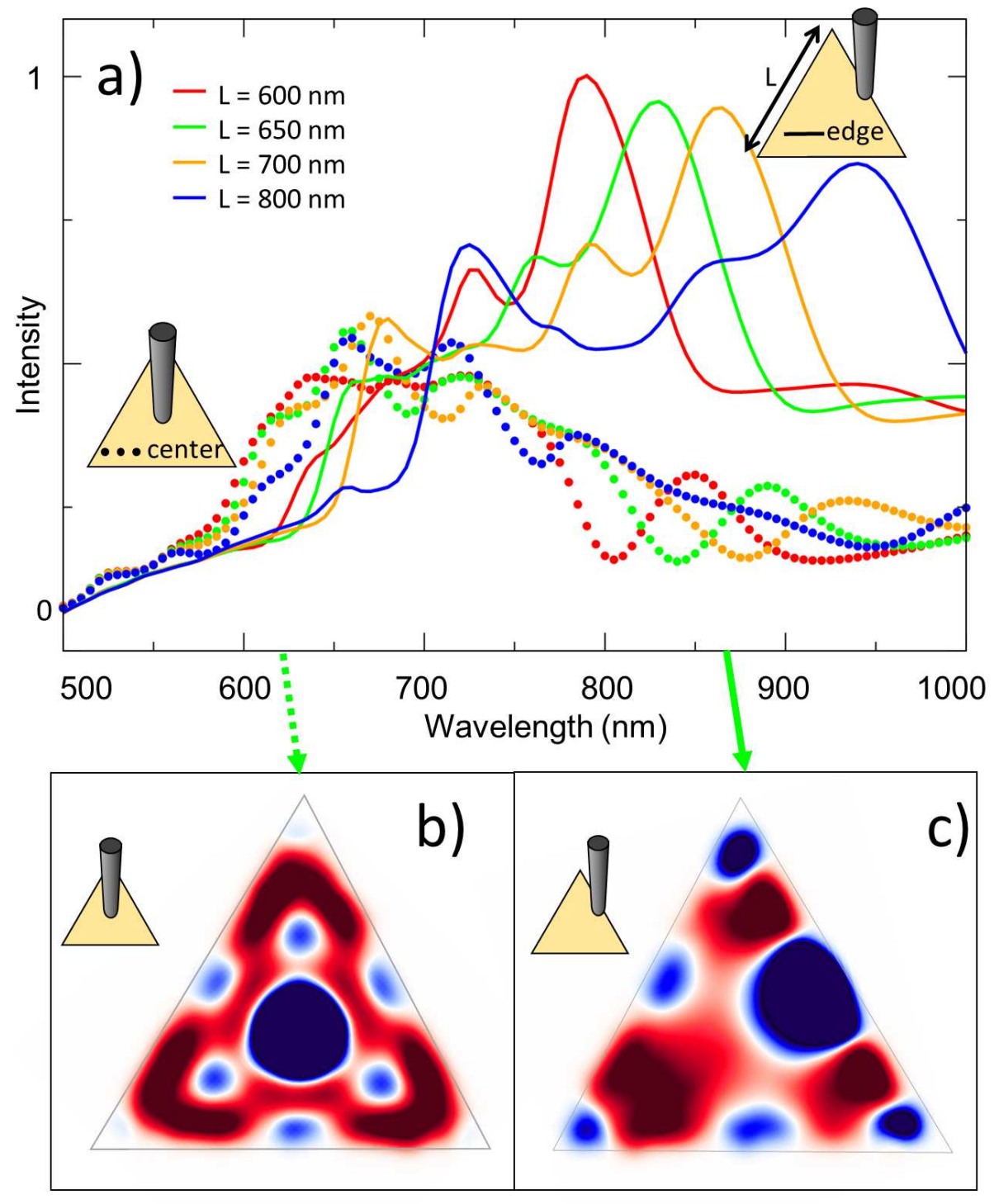

Figure 5: Calculated light emission spectra for different excitation positions and triangular platelet sizes. (a) Calculated spectra. Dotted lines: results for the STM tip located in the center of the triangle face. Continuous curves: results for the STM tip located in the center of the triangle edge. The platelets side lengths are varied from $L=600 \mathrm{~nm}$ to $L=800 \mathrm{~nm}$, as indicated in the inset. (b) and (c): surface charge density induced at a wavelength of (b) $620 \mathrm{~nm}$ and (c) $860 \mathrm{~nm}$ for the tip location indicated in the insets. The calculations are performed with a tip (radius $40 \mathrm{~nm}$, length $400 \mathrm{~nm}$ ) placed $2 \mathrm{~nm}$ above the platelet surface (substrate index of refraction is 2 ). 
R-EM-LDOS and the emission into the substrate; and (iii) the excitation of the different types of modes as a function of tip position. While the peaks in the simulated spectra are narrower than in the experiment, probably due to the idealization of the system used for the calculations (see Section 6 in the SI), the same general trend emerges from the calculated and measured data.

Figure 5b and Figure 5c display the calculated surface charge densities for the center and edge tip excitation positions at two specific energies $(\lambda=620 \mathrm{~nm}$ and $\lambda=860 \mathrm{~nm}$ respectively). As for Figure 5a, the differences in the calculation as compared to the insets of Figure 4 involve the inclusion of the STM tip and the STM-nanosource excitation spectrum, and the exclusion of the light emitted above the substrate. Once again, good agreement between the R-EM-LDOS (insets to Figure 4) and the STM excitation calculations (Figure 5b and c) is seen: at low energies and excitation on the edge (Figure 5c), the result is reminiscent of an edge mode. When the excitation is at higher energy and in the center of the platelet (Figure 5b) the resulting symmetric surface charge distribution resembles that of a breathing mode.

As pointed out previously, in the light emission experiments from an STM-nanosource, light is detected in the far field, thus this technique is expected to probe the radiative EMLDOS of the sample under study. In order to confirm this, interpret the experimental results of Figure 3 and in particular, test for possible tip or substrate effects which might perturb the link between the R-EM-LDOS and the results of the STM experiments, we perform calculations of the total EM-LDOS, of the R-EM-LDOS, and of the photon emission as a function of position for selected wavelengths. Each selected wavelength corresponds to the excitation of a different plasmonic mode of the platelet.

From the calculated total EM-LDOS, R-EM-LDOS and intensity maps in Figure 6 it follows that for longer wavelengths $(820-1300 \mathrm{~nm})$, the edge plasmon modes are predominantly excited, as seen from the intense bright spots along the edges and vertices of the platelet. The strong radiative nature of the excited edge modes explains the close similarity between 
the EM-LDOS, R-EM-LDOS and intensity maps at these wavelengths. The dominance of the radiative edge modes is also behind the good agreement between the calculations and the STM and EELS measurements at longer wavelengths (see Figure $3 \mathrm{~b}$ and d).

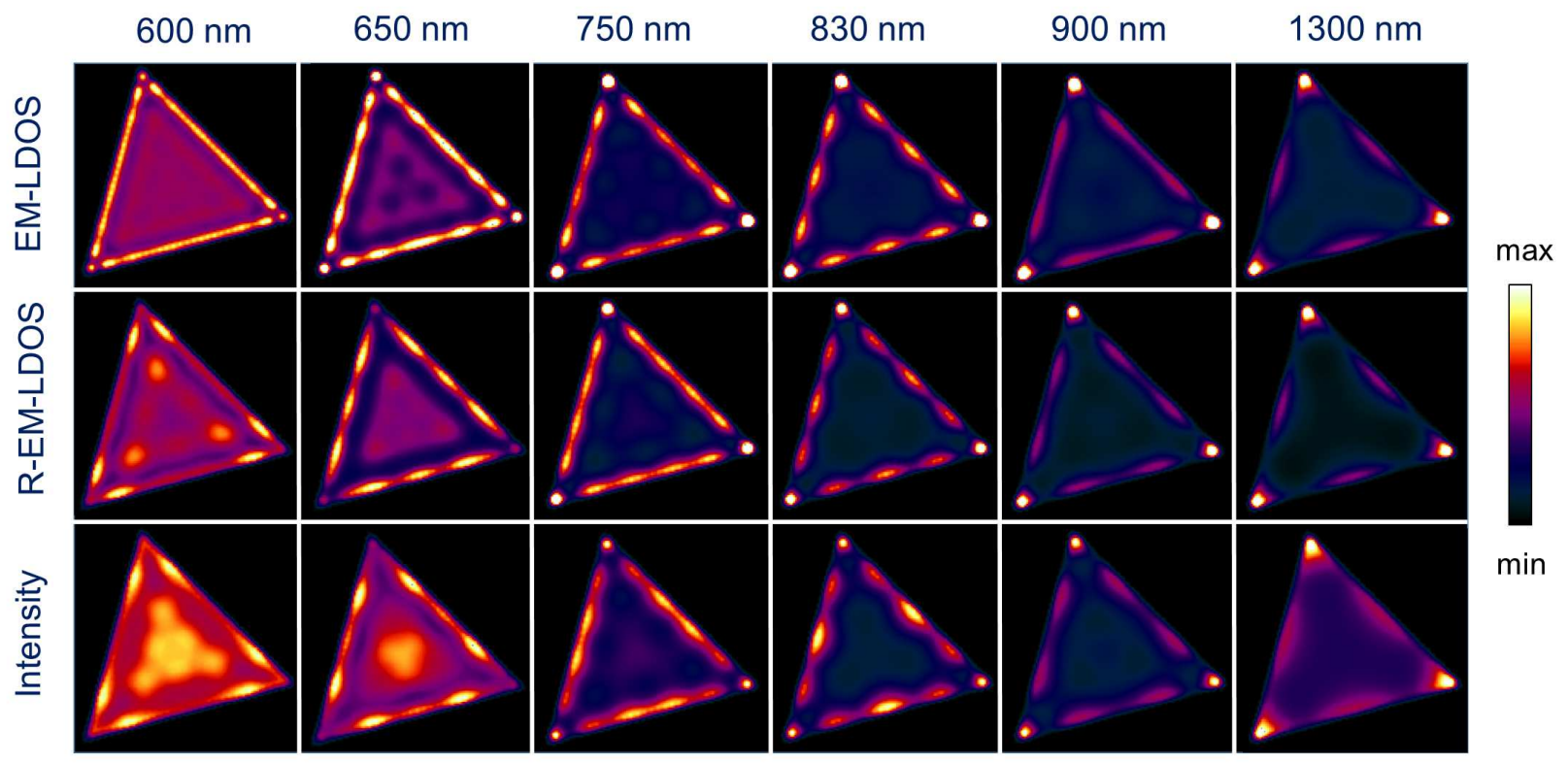

Figure 6: Calculated energy-resolved maps of the EM-LDOS, R-EM-LDOS, and of the intensity of the light emitted by the tunneling STM-nanosource when scanned over a triangular platelet with side length $L=650 \mathrm{~nm}$. The EM-LDOS is calculated $1 \mathrm{~nm}$ above the upper face of the platelet. The STM tip scans the upper face of the platelet at a constant tipsurface distance of $2 \mathrm{~nm}$. Different columns correspond to the maps obtained for different radiation wavelengths as indicated.

As the wavelength decreases from 750 to $600 \mathrm{~nm}$, the signal in the center of the maps increases, reflecting the increasing role of the breathing modes (see the left-hand side of Figure 6). This is in full agreement with the STM and EELS measurements of Figure 3a and Figure 3c. Comparing the R-EM-LDOS and EM-LDOS data in the leftmost columns of Figure 6 we observe i) a different structure in the center; ii) different relative intensities between the center and edges and iii) a different number of nodes along the edge. This is due to the fact that there are many different higher-order modes at these short wavelengths, and that these different modes contribute differently to the R-EM-LDOS and EM-LDOS. Note that such subtle differences are not clearly discernible in Figure 3 (too small signal-to-noise in the experimental data). 
The third row of Figure 6 shows the calculated photon emission maps (i.e., the emission into the substrate taking into account the presence of the tip and the STM-excitation spectrum). These results match the radiative R-EM-LDOS: the number of nodes along the triangle edges as well as the structure in the center of the platelet are well reproduced in both types of images. Note, however, that the relative intensities of the different contributions are not always the same in both cases. This is attributed to the presence of the STM tip. While it has been shown that a non-plasmonic tip does not perturb the energy spectrum of the inherent modes of the particle (see Figures 4 and 5, or Sections 1 and 5 in the Supplementary Information), it appears that the tip alters the mode coupling to the far field, as seen from the increased intensity from the center of the photon emission maps. In particular, the tip enhances the far-field coupling of the numerous breathing modes at short wavelengths.

\section{Summary and Conclusions}

In summary, we have used the inelastic tunneling current from a scanning tunneling microscope working in air to locally and electrically excite the plasmonic modes of a triangular gold platelet. The light resulting from the radiative decay of these plasmonic modes is collected through the transparent substrate. Both tip position-dependent light emission spectra as well as emission maps have been acquired, revealing that breathing modes of higher energy are more efficiently excited when the STM tip is positioned in the center of the upper face of the platelet, while edge modes at lower energies are preferably excited when the STM tip is positioned on an edge. Acquiring EELS maps on similar triangular platelets yields qualitatively similar results. The theoretical study of STM-induced light emission reproduces the main experimental trends and confirms the excitation of breathing and edge modes of the nanoparticle, associated with particular tip positions and emission energies. More importantly, a detailed comparison of the calculated total EM-LDOS and 
the radiative R-EM-LDOS reveals subtle differences in the corresponding mapping of each mode, depending on its radiative nature. Thus the scanning tunneling microscope may be considered not only a tool for mapping the electronic density of states in its usual operation mode, but also for investigating the radiative electromagnetic local density of states when the emitted light is collected. This work introduces a new technique for probing the radiative R-EM-LDOS and may be applied to obtain relevant information on nanoantennas, ${ }^{68-71}$ improved nanoplatforms for surface-enhanced Raman scattering (SERS), ${ }^{72-74}$ surface-enhanced fluorescence, ${ }^{75,76}$ single photon emission, ${ }^{71}$ and strong coupling in hybrid plasmonic-emitter systems. ${ }^{77}$

\section{Methods}

Samples: The triangular gold platelets (mono-twinned gold crystals, side length $L=$ $600-900 \mathrm{~nm}$, thickness $20 \pm 1 \mathrm{~nm}$ ) are synthesized by colloidal chemistry. A modified seedmediated growth protocol, initially proposed by Mirkin's group, is used with cetyltrimethylammonium bromide (CTAB) as the stabilizing and structure-directing agent. ${ }^{78}$ Here, contrary to the referenced protocol, the CTAB concentration is increased by a factor of $2(0.1 \mathrm{M}$ instead of $0.05 \mathrm{M}$ ), so that a mixture of thicker triangular and hexagonal platelets is obtained (20 nm instead of $9 \mathrm{~nm}$ ). A seed solution is first prepared by adding an ice-cold $\mathrm{NaBH}_{4}$ solution $(0.42 \mathrm{~mL}, 10 \mathrm{mM})$ into an aqueous solution composed of a mixture of $\mathrm{HAuCl}_{4}(92 \mu \mathrm{L}$, $10 \mathrm{mM})$ and CTAB (7 mL, $100 \mathrm{mM})$. The growth solution is prepared by successively mixing $7.3 \mathrm{~mL}$ of a $1 \mathrm{M} \mathrm{CTAB} / 50 \mu \mathrm{M} \mathrm{NaI}$ solution with $195 \mu \mathrm{l}$ of $\mathrm{HAuCl}_{4}(0.01 \mathrm{M}), 280 \mu \mathrm{l}$ of ascorbic acid $(0.016 \mathrm{M})$ and finally $43 \mu \mathrm{l}$ of $\mathrm{NaOH}(0.1 \mathrm{M})$. Adding the seeds $(2 \mu \mathrm{l}$ of the 100-fold diluted seed solution) initiates the particle synthesis reaction, which occurs for 5 hours at $40^{\circ} \mathrm{C}$. The platelets are then purified through selective sedimentation at the bottom

of the reaction vessel owing to a depletion-induced interaction. ${ }^{79}$ The chemicals used are cetyltrimethylammonium bromide $(\mathrm{CTAB} \geq 98 \%)$, chloroauric acid $\left(\mathrm{HAuCl}_{4} \cdot 3 \mathrm{H}_{2} \mathrm{O}\right)$, sodium 
borohydride $\left(\mathrm{NaBH}_{4}, 99 \%\right)$, ascorbic acid $(99 \%)$ and sodium iodide NaI (99\%). They are purchased from Sigma and used as received. Deionized water is used for all experiments.

The substrates used are either $170 \mu \mathrm{m}$ thick glass coverslips covered with an $85 \mathrm{~nm}$ thick indium-tin-oxide (ITO) layer (SOLEMS, Palaiseau, France) or $15 \mathrm{~nm}$ thick transmission electron microscope silicon nitride membrane window grids (Ted Pella). The final samples are prepared by depositing a diluted drop of the Au platelet solution on the substrate. After drying, the sample is copiously washed with ethanol, dried, and then cleaned by UV/ozone to remove any organic residue.

STM / optical microscopy setup: The commercial STM head is from JPK Instruments (NanoWizard 3). STM tips are made of tungsten using electrochemical etching (typical radii $\sim 35 \mathrm{~nm}$ ). The $\mathrm{STM}$ is mounted on top of an inverted optical microscope (Nikon Eclipse Ti-U) equipped with an oil immersion objective (Nikon CFI Apochromat 100X 1.49NA TIRF objective). The resulting light from the STM-excitation experiments is collected through the transparent substrate. For the spectral measurements, a real plane image of the sample is projected onto the slit opening of a spectrometer and integrated for $300 \mathrm{~s}$ (STM parameters are $I_{\text {set-point }}=0.5 \mathrm{nA}$ and $\left.V=2.8 \mathrm{~V}\right)$. All reported spectra have been corrected for system response. The imaging spectrometer is from Horiba Jobin Yvon (iHR320 spectrometer with a Synapse CCD detector). For the mapping, all the light from a $250 \mu \mathrm{m}^{2}$ area is detected by an avalanche photodiode (from Excelitas, dark count as low as 5 counts/s) and integrated for each pixel by the STM controller. A bandpass filter (center wavelength $750 \mathrm{~nm}$ and bandwidth $40 \mathrm{~nm}$, or 900-nm center wavelength and again a bandwidth of $40 \mathrm{~nm}$ ) is placed before the detector. As a result, the topography image, tunneling current map and photon map for a particular wavelength range may be acquired simultaneously. In the STM experiments a (positive) voltage is applied to the sample and the tip is grounded. This voltage is either $0.5 \mathrm{~V}$ for imaging or $2.8 \mathrm{~V}$ for excitation (a voltage of $>1.5 \mathrm{~V}$ is necessary for emission in the visible). These measurements are performed in constant current mode with a feedback loop which maintains the measured tunneling current nominally constant at a value of $0.1 \mathrm{nA}$. 
Note that triangle side lengths $L$ measured by STM are most likely overestimated due to convolution with the tip. All of the reported measurements are carried out in air under standard laboratory conditions. As a result, a thin water layer exists on both the sample and the tip. Details on the specificities of the STM-excitation of plasmons in air may be found in the reference Rogez et al. ${ }^{80}$.

Electron energy loss spectroscopy: STEM-EELS measurements are performed using a Vacuum Generator HB501 scanning transmission electron microscope (STEM) operated at $100 \mathrm{kV}$, fitted with a cold field emission gun and a homemade electron energy loss spectrometer (EELS) detection system. The electron probe size is a few nanometers and the EEL spectrometer energy dispersion is $20 \mathrm{meV} /$ channel. The data is collected in the spectral image mode where high-angle annular dark field (HAADF) signals and EEL spectra are collected simultaneously pixel by pixel while scanning. At the end of a scan, both a HAADF image and a spectrum-image (EEL spectrum at each pixel of the scan) are generated which can be compared pixel by pixel. By means of a spectrum-image, filtered maps are created by plotting the EEL signal intensity of each pixel in a chosen energy range. Typical acquisition times per pixel are $5 \mathrm{~ms}$ and spectral image data sets are 100 x 100 pixels large. Spectral alignment and Richardson-Lucy deconvolution are used in order to improve the energy resolution from $\sim 0.3 \mathrm{eV}$ to $\sim 0.1 \mathrm{eV}$ (zero loss peak full-width at half maximum) using the Hyperspy software. ${ }^{81}$

Simulations: Three-dimensional electrodynamic calculations are performed for a unitary harmonic point source emitter with current density given by $\vec{j}=\hat{u} \delta\left(\vec{r}-\overrightarrow{r_{0}}\right)$, where the unitary vector $\hat{u}$ defines the polarization of the source. The position $\overrightarrow{r_{0}}$ of the point source is $1 \mathrm{~nm}$ above the upper face of the platelet for the calculations. In the case of the photon maps, an STM tip is also included $1 \mathrm{~nm}$ above the point source. Unless otherwise stated, we consider that the tunneling source is oriented perpendicularly to the platelet face along the symmetry axis of the tip. The finite element method as implemented in the frequency domain using the radio frequency module of the commercial COMSOL Multiphysics software ${ }^{82}$ 
is used. For the photon emission spectral calculations, the resulting calculated spectra are multiplied by a factor of $(e V-\hbar \omega)$ (where $e$ is the charge of an electron, $V$ is the applied voltage between the tip and sample, and $\omega$ is the photon angular frequency) in order to take into account the inelastic tunneling electron probability as a function of energy. ${ }^{57,83}$ For the calculation of the EM-LDOS we consider that it is dominated by the electromagnetic modes of the platelet, and we use the expression for the change of the rate of the radiative and non-radiative energy dissipation by the point emitter due to the presence of the metal. ${ }^{84}$

$$
\Gamma=-\frac{1}{2} \operatorname{Re}\left\{\hat{u} \cdot \vec{E}\left(\overrightarrow{r_{0}}\right)\right\}
$$

where $\vec{E}\left(\overrightarrow{r_{0}}\right)$ is the electric field (at the position of the point source emitter) created by the gold platelet (the calculations are performed without an STM tip). Re $\{Z\}$ stands for the real part of the complex quantity $Z$. On the other hand, the change of the power dissipated by the unitary point source can be presented through the electromagnetic density of states of the metal, $\rho_{\hat{u}}^{m}\left(\overrightarrow{r_{0}}, \omega\right)$, as follows ${ }^{2}$

$$
\Gamma=\frac{\pi}{4 \epsilon_{0}} \rho_{\hat{u}}^{m}\left(\overrightarrow{r_{0}}, \omega\right)
$$

This immediately leads to $\rho_{\hat{u}}^{m}\left(\overrightarrow{r_{0}}, \omega\right)$ from the calculated $\operatorname{Re}\left\{\hat{u} \vec{E}\left(\overrightarrow{r_{0}}\right)\right\}$. We consider that $\rho_{\hat{u}}^{m}\left(\overrightarrow{r_{0}}, \omega\right)>>\rho_{\hat{u}, 0}\left(\overrightarrow{r_{0}}, \omega\right)$, where $\rho_{\hat{u}, 0}\left(\overrightarrow{r_{0}}, \omega\right)=\frac{\omega^{2}}{3 \pi^{2} c^{3}}$ is the projected EM-LDOS in vacuum $(\omega$ is the angular frequency and $c$ is the speed of light).

For the R-EM-LDOS mapping results, the Poynting vector in the far field is integrated over a full sphere enclosing the system (the calculations are performed without an STM tip). For the photon emission spectra and photon emission maps the calculations are performed for the unitary harmonic point source emitter placed in the junction between the STM tip and platelet surface, and the Poynting vector in the far field is integrated over a half sphere beneath the substrate.

The gold triangle (20 $\mathrm{nm}$ thick) is considered equilateral with rounded vertices (radius 
of curvature $5 \mathrm{~nm}$ ). Side lengths of 600 to $900 \mathrm{~nm}$ are considered. The index of refraction

of gold that is used is from Johnson and Christy. ${ }^{85}$ The substrate is represented either by a semi-infinite space with refraction index $n=2$ below the triangle structure, or, in the case of the surface charge density calculations of Figure 5, by a disk of radius $1600 \mathrm{~nm}$ and of thickness $200 \mathrm{~nm}$ (index of refraction is 2), with air above and below. The full environment is a sphere of radius $2000 \mathrm{~nm}$, surrounded by a 300 or $600 \mathrm{~nm}$ thick perfectly matched layer. The tungsten tip (index of refraction from Rakić et al. ${ }^{60}$ ) is represented by a solid cone which has an opening angle of $\tan \theta=\frac{1}{6}$ and whose end is replaced by a sphere of radius $40 \mathrm{~nm}$. The length of the tip is $400 \mathrm{~nm}$. See Section 1 of the Supplementary Information for results with different values for the tip radius and length.

\section{Acknowledgement}

S.C. acknowledges the financial support of the China Scholarship Council (CSC) (No. 201304910386).

M. Z.-H. acknowledges the financial support of the European Union under the Project H2020 FETOPEN-2016-2017 "FEMTOTERABYTE" (Project n 737093) and the "Colombian Administrative Department of Science, Technology and Innovation" - COLCIENCIAS under its "Estancias Postdoctorales 784-2017" call. He also acknowledges the hospitality of the Institute des Sciences Moléculaires d'Orsay and Dr. Nuno de Sousa for his guidance and support using COMSOL Multiphysics. S.M. was supported by the HAPPLE grant (French National Research Agency : ANR-12-BS10-0016). This work is partially funded by the Conseil Régional, Île-de-France (DIM Nano-K). JA acknowledges Project FIS2016-80174-P of the Spanish MICINN, and project IT1164-19 of the Basque Government.

\section{Supporting Information Available}

1. Calculated spectra and tip effects 
2. STM-nanosource mapping experiments

3. Electron energy loss spectroscopy: spectra and spatially resolved maps

4. Spectra as a function of triangle side length

5. Comparison of the calculated emission above and below the substrate, and the R-EMLDOS

6. Triangle shapes and dimensions: HAADF STEM images

This material is available free of charge via the Internet at http://pubs.acs.org

\section{References}

(1) Vahala, K. J. Optical microcavities. Nature 2003, 424, 839.

(2) Carminati, R.; Caze, A.; Cao, D.; Peragut, F.; Krachmalnicoff, V.; Pierrat, R.; De Wilde, Y. Electromagnetic density of states in complex plasmonic systems. Surf. Sci. Rep. 2015, 70, 1-41.

(3) Callahan, D. M.; Munday, J. N.; Atwater, H. A. Solar Cell Light Trapping beyond the Ray Optic Limit. Nano Letters 2012, 12, 214-218.

(4) Dousse, A.; Suffczyński, J.; Beveratos, A.; Krebs, O.; Lemaître, A.; Sagnes, I.; Bloch, J.; Voisin, P.; Senellart, P. Ultrabright source of entangled photon pairs. Nature 2010, 466, 217.

(5) Hoang, T. B.; Akselrod, G. M.; Argyropoulos, C.; Huang, J.; Smith, D. R.; Mikkelsen, M. H. Ultrafast spontaneous emission source using plasmonic nanoantennas. Nat. Commun. 2015, 6, 7788. 
(6) Joulain, K.; Carminati, R.; Mulet, J.-P.; Greffet, J.-J. Definition and measurement of the local density of electromagnetic states close to an interface. Phys. Rev. B 2003, 68, 245405.

(7) Novotny, L.; Hecht, B. Principles of nano-optics; Cambridge University Press, 2006.

(8) Nelayah, J.; Gu, L.; Sigle, W.; Koch, C. T.; Pastoriza-Santos, I.; Liz-Marzán, L. M.; van Aken, P. A. Direct imaging of surface plasmon resonances on single triangular silver nanoprisms at optical wavelength using low-loss EFTEM imaging. Opt. Lett. 2009, 34, $1003-1005$.

(9) Talebi, N.; Sigle, W.; Vogelgesang, R.; Koch, C. T.; Fernández-López, C.; LizMarzán, L. M.; Ögüt, B.; Rohm, M.; van Aken, P. A. Breaking the Mode Degeneracy of Surface Plasmon Resonances in a Triangular System. Langmuir 2012, 28, 8867-8873.

(10) Kociak, M.; Stephan, O. Mapping plasmons at the nanometer scale in an electron microscope. Chem. Soc. Rev. 2014, 43, 3865-3883.

(11) Hörl, A.; Haberfehlner, G.; Trügler, A.; Schmidt, F.-P.; Hohenester, U.; Kothleitner, G. Tomographic imaging of the photonic environment of plasmonic nanoparticles. Nature Communications 2017, 8, 37.

(12) Garcia de Abajo, F. J.; Kociak, M. Probing the photonic local density of states with electron energy loss spectroscopy. Phys. Rev. Lett. 2008, 100, 106804.

(13) Hohenester, U.; Ditlbacher, H.; Krenn, J. R. Electron-Energy-Loss Spectra of Plasmonic Nanoparticles. Phys. Rev. Lett. 2009, 103, 106801.

(14) Krehl, J.; Guzzinati, G.; Schultz, J.; Potapov, P.; Pohl, D.; Martin, J.; Verbeeck, J.; Fery, A.; Buechner, B.; Lubk, A. Spectral field mapping in plasmonic nanostructures with nanometer resolution. Nat. Commun. 2018, 9, 4207. 
(15) Kuttge, M.; Vesseur, E. J. R.; Koenderink, A. F.; Lezec, H. J.; Atwater, H. A.; Garcia de Abajo, F. J.; Polman, A. Local density of states, spectrum, and far-field interference of surface plasmon polaritons probed by cathodoluminescence. Phys. Rev. B 2009, 79, 113405.

(16) Sapienza, R.; Coenen, T.; Renger, J.; Kuttge, M.; van Hulst, N. F.; Polman, A. Deepsubwavelength imaging of the modal dispersion of light. Nat. Mater. 2012, 11, 781-787.

(17) Losquin, A.; Kociak, M. Link between Cathodoluminescence and Electron Energy Loss Spectroscopy and the Radiative and Full Electromagnetic Local Density of States. ACS Photonics 2015, 2, 1619-1627.

(18) Losquin, A.; Zagonel, L. F.; Myroshnychenko, V.; Rodrguez-González, B.; Tencé, M.; Scarabelli, L.; Förstner, J.; Liz-Marzán, L. M.; García de Abajo, F. J.; Stéphan, O.; Kociak, M. Unveiling Nanometer Scale Extinction and Scattering Phenomena through Combined Electron Energy Loss Spectroscopy and Cathodoluminescence Measurements. Nano Letters 2015, 15, 1229-1237.

(19) Myroshnychenko, V.; Nishio, N.; García de Abajo, F. J.; Förstner, J.; Yamamoto, N. Unveiling and Imaging Degenerate States in Plasmonic Nanoparticles with Nanometer Resolution. ACS Nano 2018, 12, 8436-8446.

(20) Hoogenboom, J. P.; Sanchez-Mosteiro, G.; Colas des Francs, G.; Heinis, D.; Legay, G.; Dereux, A.; van Hulst, N. F. The Single Molecule Probe: Nanoscale Vectorial Mapping of Photonic Mode Density in a Metal Nanocavity. Nano Letters 2009, 9, 1189-1195.

(21) Beams, R.; Smith, D.; Johnson, T. W.; Oh, S.-H.; Novotny, L.; Vamivakas, A. N. Nanoscale Fluorescence Lifetime Imaging of an Optical Antenna with a Single Diamond NV Center. Nano Letters 2013, 13, 3807-3811.

(22) Ropp, C.; Cummins, Z.; Nah, S.; Fourkas, J. T.; Shapiro, B.; Waks, E. Nanoscale 
imaging and spontaneous emission control with a single nano-positioned quantum dot. Nature Communications 2013, 4, 1447.

(23) Krachmalnicoff, V.; Cao, D.; Caze, A.; Castanie, E.; Pierrat, R.; Bardou, N.; Collin, S.; Carminati, R.; De Wilde, Y. Towards a full characterization of a plasmonic nanostructure with a fluorescent near-field probe. Opt. Express 2013, 21, 11536-11545.

(24) Schell, A. W.; Engel, P.; Werra, J. F. M.; Wolff, C.; Busch, K.; Benson, O. Scanning Single Quantum Emitter Fluorescence Lifetime Imaging: Quantitative Analysis of the Local Density of Photonic States. Nano Letters 2014, 14, 2623-2627.

(25) Cao, D.; Caze, A.; Calabrese, M.; Pierrat, R.; Bardou, N.; Collin, S.; Carminati, R.; Krachmalnicoff, V.; De Wilde, Y. Mapping the Radiative and the Apparent Nonradiative Local Density of States in the Near Field of a Metallic Nanoantenna. ACS Photonics 2015, 2, 189-193.

(26) Aigouy, L.; Caze, A.; Gredin, P.; Mortier, M.; Carminati, R. Mapping and Quantifying Electric and Magnetic Dipole Luminescence at the Nanoscale. Phys. Rev. Lett. 2014, 113, 076101.

(27) Cuche, A.; Berthel, M.; Kumar, U.; Colas des Francs, G.; Huant, S.; Dujardin, E.; Girard, C.; Drezet, A. Near-field hyperspectral quantum probing of multimodal plasmonic resonators. Phys. Rev. B 2017, 95, 121402.

(28) Viarbitskaya, S.; Teulle, A.; Marty, R.; Sharma, J.; Girard, C.; Arbouet, A.; Dujardin, E. Tailoring and imaging the plasmonic local density of states in crystalline nanoprisms. Nat. Mater. 2013, 12, 426-432.

(29) Imaeda, K.; Hasegawa, S.; Imura, K. Static and Dynamic Near-Field Measurements of High-Order Plasmon Modes Induced in a Gold Triangular Nanoplate. J. Phys. Chem. Lett. 2018, 9, 4075-4081. 
(30) Chicanne, C.; David, T.; Quidant, R.; Weeber, J. C.; Lacroute, Y.; Bourillot, E.; Dereux, A.; Colas des Francs, G.; Girard, C. Imaging the Local Density of States of Optical Corrals. Phys. Rev. Lett. 2002, 88, 097402.

(31) Rang, M.; Jones, A. C.; Zhou, F.; Li, Z.-Y.; Wiley, B. J.; Xia, Y.; Raschke, M. B. Optical Near-Field Mapping of Plasmonic Nanoprisms. Nano Lett. 2008, 8, 3357-3363.

(32) Imaeda, K.; Hasegawa, S.; Imura, K. Imaging of Plasmonic Eigen Modes in Gold Triangular Mesoplates by Near-Field Optical Microscopy. J. Phys. Chem. C 2018, 122, 7399-7409.

(33) Awada, C.; Popescu, T.; Douillard, L.; Charra, F.; Perron, A.; Yockell-Lelièvre, H.; Baudrion, A.-L.; Adam, P.-M.; Bachelot, R. Selective Excitation of Plasmon Resonances of Single Au Triangles by Polarization-Dependent Light Excitation. J. Phys. Chem. C 2012, 116, 14591-14598.

(34) Frank, B.; Kahl, P.; Podbiel, D.; Spektor, G.; Orenstein, M.; Fu, L.; Weiss, T.; Horn-von Hoegen, M.; Davis, T. J.; Meyer zu Heringdorf, F.-J.; Giessen, H. Short-range surface plasmonics: Localized electron emission dynamics from a 60-nm spot on an atomically flat single-crystalline gold surface. Science Advances 2017, 3, e1700721.

(35) Tersoff, J.; Hamann, D. R. Theory of the scanning tunneling microscope. Phys. Rev. B 1985, 31, 805-813.

(36) Schull, G.; Becker, M.; Berndt, R. Imaging Confined Electrons with Plasmonic Light. Phys. Rev. Lett. 2008, 101, 136801.

(37) Gimzewski, J. K.; Sass, J. K.; Schlitter, R. R.; Schott, J. Enhanced Photon-emission In Scanning Tunnelling Microscopy. Europhysics Letters 1989, 8, 435-440.

(38) Wang, T.; Boer-Duchemin, E.; Zhang, Y.; Comtet, G.; Dujardin, G. Excitation of 
propagating surface plasmons with a scanning tunnelling microscope. Nanotechnology 2011, 22, 175201.

(39) Wang, T.; Boer-Duchemin, E.; Comtet, G.; Le Moal, E.; Dujardin, G.; Drezet, A.; Huant, S. Plasmon scattering from holes: from single hole scattering to Young's experiment. Nanotechnology 2014, 25, 125202.

(40) Bharadwaj, P.; Bouhelier, A.; Novotny, L. Electrical Excitation of Surface Plasmons. Phys. Rev. Lett. 2011, 106, 226802.

(41) Myrach, P.; Nilius, N.; Freund, H.-J. Photon mapping of individual Ag particles on $\mathrm{MgO} / \mathrm{Mo}(001)$. Phys. Rev. B 2011, 83, 035416.

(42) Yu, A.; Li, S.; Czap, G.; Ho, W. Tunneling-Electron-Induced Light Emission from Single Gold Nanoclusters. Nano Lett. 2016, 16, 5433-5436.

(43) Kuhnke, K.; Große, C.; Merino, P.; Kern, K. Atomic-Scale Imaging and Spectroscopy of Electroluminescence at Molecular Interfaces. Chem. Rev. 2017, 117, 5174-5222.

(44) Leon, C. C.; Rosławska, A.; Grewal, A.; Gunnarsson, O.; Kuhnke, K.; Kern, K. Photon superbunching from a generic tunnel junction. Science Advances 2019, 5, eaav4986.

(45) Martín-Jiménez, A.; Fernández-Domínguez, A. I.; Lauwaet, K.; Granados, D.; Miranda, R.; García-Vidal, F. J.; Otero, R. Unveiling the radiative local density of optical states of a plasmonic nanocavity by STM. Nature Communications 2020, 11, 1021.

(46) Le Moal, E.; Marguet, S.; Rogez, B.; Mukherjee, S.; Dos Santos, P.; Boer-Duchemin, E.; Comtet, G.; Dujardin, G. An electrically excited nanoscale light source with active angular control of the emitted light. Nano Letters 2013, 13, 4198-205.

(47) Cao, S. Y.; Le Moal, E.; Jiang, Q. B.; Drezet, A.; Huant, S.; Hugonin, J. P.; Dujardin, G.; Boer-Duchemin, E. Directional light beams by design from electrically driven elliptical slit antennas. Beilstein Journal of Nanotechnology 2018, 9, 2361-2371. 
(48) Kern, J.; Kullock, R.; Prangsma, J.; Emmerling, M.; Kamp, M.; Hecht, B. Electrically driven optical antennas. Nature Photonics 2015, 9, 582.

(49) Du, W.; Wang, T.; Chu, H.-S.; Nijhuis, C. A. Highly efficient on-chip direct electronicplasmonic transducers. Nature Photonics 2017, 11, 623.

(50) Gurunarayanan, S. P.; Verellen, N.; Zharinov, V. S.; Shirley, F. J.; Moshchalkov, V. V.; Heyns, M.; Van de Vondel, J.; Radu, I. P.; Van Dorpe, P. Electrically Driven Unidirectional Optical Nanoantennas. Nano Letters 2017, 17, 7433-7439.

(51) Qian, H.; Hsu, S.-W.; Gurunatha, K.; Riley, C. T.; Zhao, J.; Lu, D.; Tao, A. R.; Liu, Z. Efficient light generation from enhanced inelastic electron tunnelling. Nature Photonics 2018, 12, 485 .

(52) Parzefall, M.; Novotny, L. Optical antennas driven by quantum tunneling: a key issues review. Reports on Progress in Physics 2019, 82, 112401.

(53) Zhang, C.; Hugonin, J.-P.; Coutrot, A.-L.; Sauvan, C.; Marquier, F.; Greffet, J.-J. Antenna surface plasmon emission by inelastic tunneling. Nature Communications 2019, 10, 4949 .

(54) Johansson, P. Light emission from a scanning tunneling microscope: Fully retarded calculation. Phys. Rev. B 1998, 58, 10823-10834.

(55) Uehara, Y.; Kimura, Y.; Ushioda, S.; Takeuchi, K. Theory of Visible-light Emission From Scanning Tunneling Microscope. Japanese Journal of Applied Physics Part 1regular Papers Short Notes \& Review Papers 1992, 31, 2465-2469.

(56) Aizpurua, J.; Apell, S. P.; Berndt, R. Role of tip shape in light emission from the scanning tunneling microscope. Phys. Rev. B 2000, 62, 2065-2073.

(57) Lambe, J.; McCarthy, S. L. Light Emission from Inelastic Electron Tunneling. Phys. Rev. Lett. 1976, 37, 923-925. 
(58) Le Moal, E.; Marguet, S.; Canneson, D.; Rogez, B.; Boer-Duchemin, E.; Dujardin, G.; Teperik, T. V.; Marinica, D.-C.; Borisov, A. G. Engineering the emission of light from a scanning tunneling microscope using the plasmonic modes of a nanoparticle. Phys. Rev. B 2016, 93, 035418.

(59) Mitra, J.; Feng, L.; Boyle, M. G.; Dawson, P. Electromagnetic interaction between a metallic nanoparticle and surface in tunnelling proximity-modelling and experiment. J. Phys. D-Appl. Phys. 2009, 42, 215101.

(60) Rakić, A. D.; Djurišić, A. B.; Elazar, J. M.; Majewski, M. L. Optical properties of metallic films for vertical-cavity optoelectronic devices. Appl. Opt. 1998, 37, 52715283.

(61) Schmidt, F.-P.; Ditlbacher, H.; Hohenester, U.; Hohenau, A.; Hofer, F.; Krenn, J. R. Universal dispersion of surface plasmons in flat nanostructures. Nature Communications 2014, 5, 3604.

(62) Campos, A.; Arbouet, A.; Martin, J.; Gérard, D.; Proust, J.; Plain, J.; Kociak, M. Plasmonic Breathing and Edge Modes in Aluminum Nanotriangles. ACS Photonics 2017, 4, 1257-1263.

(63) Keast, V. J.; Walhout, C.; Pedersen, T. B.; Shahcheraghi, N.; Cortie, M. B.; Mitchell, D. R. G. Higher Order Plasmonic Modes Excited in Ag Triangular Nanoplates by an Electron Beam. Plasmonics 2015, 11, 1081-1086.

(64) Schmidt, F.-P.; Ditlbacher, H.; Hohenester, U.; Hohenau, A.; Hofer, F.; Krenn, J. R. Dark Plasmonic Breathing Modes in Silver Nanodisks. Nano Letters 2012, 12, 57805783.

(65) Krug, M. K.; Reisecker, M.; Hohenau, A.; Ditlbacher, H.; Truegler, A.; Hohenester, U.; Krenn, J. R. Probing plasmonic breathing modes optically. Applied Physics Letters 2014, 105, 171103. 
(66) Schmidt, F.-P.; Losquin, A.; Hofer, F.; Hohenau, A.; Krenn, J. R.; Kociak, M. How Dark Are Radial Breathing Modes in Plasmonic Nanodisks? ACS Photonics 2018, 5, 861-866.

(67) Bicket, I. C.; Bellido, E. P.; McRae, D. M.; Lagugne-Labarthet, F.; Botton, G. A. Carving Plasmon Modes in Silver Sierpinski Fractals. ACS Photonics 2019, 6, 29742984.

(68) Novotny, L.; van Hulst, N. Antennas for light. Nat. Photonics 2011, 5, 83-90.

(69) Giannini, V.; Fernandez-Dominguez, A. I.; Heck, S. C.; Maier, S. A. Plasmonic Nanoantennas: Fundamentals and Their Use in Controlling the Radiative Properties of Nanoemitters. Chem. Rev. 2011, 111, 3888-3912.

(70) Biagioni, P.; Huang, J.-S.; Hecht, B. Nanoantennas for visible and infrared radiation. Rep. Prog. Phys. 2012, 75.

(71) Koenderink, A. F. Single-Photon Nanoantennas. ACS Phot. 2017, 4, 710-722.

(72) Kleinman, S. L.; Frontiera, R. R.; Henry, A.-I.; Dieringer, J. A.; Van Duyne, R. P. Creating, characterizing, and controlling chemistry with SERS hot spots. Phys. Chem. Chem. Phys. 2013, 15, 21-36.

(73) Zhang, R.; Zhang, Y.; Dong, Z. C.; Jiang, S.; Zhang, C.; Chen, L. G.; Zhang, L.; Liao, Y.; Aizpurua, J.; Luo, Y.; Yang, J. L.; Hou, J. G. Chemical mapping of a single molecule by plasmon-enhanced Raman scattering. Nature 2013, 498, 82-86.

(74) Lee, J.; Crampton, K. T.; Tallarida, N.; Apkarian, V. A. Visualizing vibrational normal modes of a single molecule with atomically confined light. Nature 2019, 568, 78-82.

(75) Dong, Z. C.; Zhang, X. L.; Gao, H. Y.; Luo, Y.; Zhang, C.; Chen, L. G.; Zhang, R.; Tao, X.; Zhang, Y.; Yang, J. L.; Hou, J. G. Generation of molecular hot electroluminescence by resonant nanocavity plasmons. Nature Photonics 2010, 4, 50-54. 
(76) Doppagne, B.; Neuman, T.; Soria-Martinez, R.; López, L. E. P.; Bulou, H.; Romeo, M.; Berciaud, S.; Scheurer, F.; Aizpurua, J.; Schull, G. Single-molecule tautomerization tracking through space- and time-resolved fluorescence spectroscopy. Nature Nanotechnology 2020, 15, 207-211.

(77) Törma, P.; Barnes, W. L. Strong coupling between surface plasmon polaritons and emitters: a review. Rep. Prog. Phys. 2015, 78, 013901.

(78) Young, K. L.; Jones, M. R.; Zhang, J.; Macfarlane, R. J.; Esquivel-Sirvent, R.; Nap, R. J.; Wu, J.; Schatz, G. C.; Lee, B.; Mirkin, C. A. Assembly of reconfigurable onedimensional colloidal superlattices due to a synergy of fundamental nanoscale forces. Proceedings of the National Academy of Sciences 2012, 109, 2240-2245.

(79) Straney, P. J.; Marbella, L. E.; Andolina, C. M.; Nuhfer, N. T.; Millstone, J. E. Decoupling Mechanisms of Platinum Deposition on Colloidal Gold Nanoparticle Substrates. J. Am. Chem. Soc. 2014, 136, 7873-7876.

(80) Rogez, B.; Cao, S.; Dujardin, G.; Comtet, G.; Le Moal, E.; Mayne, A.; BoerDuchemin, E. The mechanism of light emission from a scanning tunnelling microscope operating in air. Nanotechnology 2016, 27, 465201.

(81) HyperSpy 1.0. https://doi.org/10.5281/zenodo.57882.

(82) COMSOL Multyphisics v. 5.2, AB, Stockholm, Sweden. www.comsol.com.

(83) Persson, B. N. J.; Baratoff, A. Theory of Photon-emission In Electron-tunneling To Metallic Particles. Phys. Rev. Lett. 1992, 68, 3224-3227.

(84) Chen, S.; Autore, M.; Li, J.; Li, P.; Alonso-Gonzalez, P.; Yang, Z.; Martin-Moreno, L.; Hillenbrand, R.; Nikitin, A. Y. Acoustic Graphene Plasmon Nanoresonators for FieldEnhanced Infrared Molecular Spectroscopy. ACS Photonics 2017, 4, 3089-3097. 
(85) Johnson, P. B.; Christy, R. W. Optical Constants of the Noble Metals. Phys. Rev. B 1972, 6, 4370-4379. 


\section{Graphical TOC Entry}

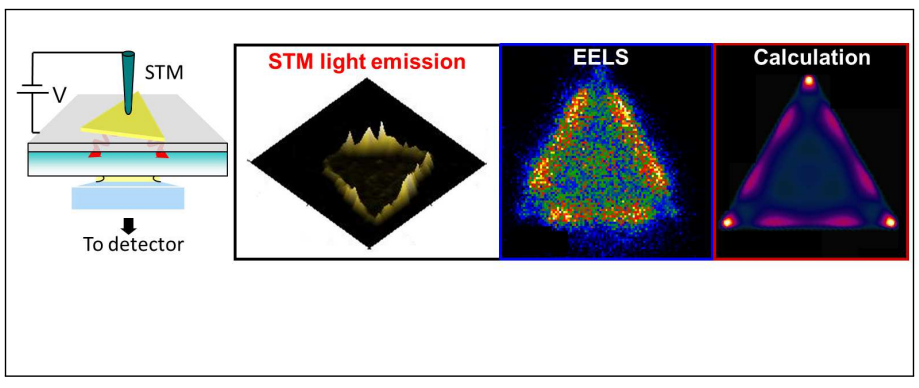

For Table of Contents Use Only

Article title: Probing the radiative electromagnetic local density of states in nanostructures with a scanning tunneling microscope.

Authors: Shuiyan Cao, Mario Zapata-Hererra, Alfredo Campos, Eric Le Moal, Sylvie Marguet, Gérald Dujardin, Mathieu Kociak, Javier Aizpurua, Andrei G. Borisov, and Elizabeth Boer-Duchemin

Description of TOC entry: (leftmost image): In this work, a scanning tunneling microscope (STM) is used to locally and electrically excite the electromagnetic modes of a triangular gold platelet. The modes decay radiatively and the emitted light is detected through the transparent substrate in the far field. (center left): When the STM tip is located on the edge of the platelet, the emission is strongest. These STM results are shown to agree well with electron energy loss spectroscopy (EELS) (center right) and calculations (rightmost image), leading to the conclusion that the STM-nanosource may be considered a local probe of the radiative local density of electromagnetic states. 